B. Buffel ${ }^{\text {* }}$, B. van Mieghem ${ }^{2}$, A. van Bael ${ }^{2}$, F. Desplentere ${ }^{1}$

${ }^{1}$ KU Leuven Technology Campus Oostende, Oostende, Belgium

${ }^{2}$ KU Leuven Technology Campus Diepenbeek, Diepenbeek, Belgium

\title{
A Combined Experimental and Modelling Approach towards an Optimized Heating Strategy in Thermoforming of Thermoplastics Sheets
}

\begin{abstract}
Determining the operational settings for the heating equipment in thermoforming is still mainly done by trial and error as well as personal experience. Depending on the type of $I R$ heating equipment, these settings can be the consumed electrical power or the desired temperature of the heating elements. In this study, a workflow is developed, applied and validated to characterize the IR heating equipment and to determine the optimal heating strategy. The workflow starts with an onsite equipment/machine characterization, which takes all machine and environment parameters into account. This approach results in the optimal heater setting and heating duration in order to obtain a through thickness temperature distribution which lies within a predefined forming range. The proposed methodology is universally applicable as it can deal with different types of sheet material and thicknesses. Moreover it can be applied to any type of IR heating element (halogen, metal foil, ceramic or quartz). Moreover, the methodology can easily be implemented in an industrial environment. Additionally, an estimate for the thermal efficiency of halogen heater equipment can be determined.
\end{abstract}

\section{Introduction}

Thermoforming of thermoplastic sheets is a versatile process in which both thin $(<1 \mathrm{~mm})$ and thick $(>1 \mathrm{~mm})$ sheets (Van Mieghem, 2015) are transformed into consumer goods or technical products. Based on the achievable production rate, thin gauge thermoforming is able to compete with injection molding, while thick gauge thermoforming is more dedicated to smaller production volumes (max. 10000 parts) and products with specific demands or with integrated functionalities (e.g. metal inserts, textile surface finishing).

Thermoforming consists of three main steps: (1) heating of the sheet by means of contact, convective and/or radiative heat transfer, (2) forming of the product in or on a mold and (3) cooling and demolding of the part (Throne, 1996). A key fea-

* Mail address: Bart Buffel, KU Leuven Technology Campus Oostende, Zeedijk 101, 8400 Oostende, Belgium

E-mail: bart.buffel@kuleuven.be ture of the process is a non-uniform thickness distribution in the final part, which is caused by different draw ratios and inplane temperature variations. An optimized heating strategy and sheet temperature is therefore imperative to obtain a qualitative product, which fulfils the customers' demands (Yousefi et al., 2002).

In practice the heating of the sheet is mainly done by means of infrared radiation via ceramic, quartz, halogen or metal foil heater elements. The use of different settings of these heater elements allow an in-plane temperature distribution of the sheet which is obtained by using a grid of heating elements with different settings (Duarte et al., 2004; Lee et al., 2001a). The absorption of the radiative heat combined with the low thermal conductivity of thermoplastic materials, leads to a varying temperature distribution through thickness which is nearly inevitable and should be controlled. Next to the temperature evolution of the thermoplastic sheet, also the temperature of the mold and possible assisting plugs influence the final thickness distribution of the product (Marathe et al., 2016). Despite the large influence of the sheet temperature on the part quality (Lee et al., 2001b), process start-up and control is still mainly based on trial and error as well as personal experience. This leads to varying machine settings, excessive material waste and high start-up times and costs. There is thus a need for a higher level of process monitoring and control (Gilham, 2016). The present study focuses on the characterization and optimization of the through-thickness distribution. In order to be industrially relevant, the heating should be accomplished as quickly as possible without producing temperature variations exceeding the desired forming range (Throne, 1996).

Measuring and monitoring the sheet temperature during the heating phase of the process can be done by means of thermocouples or contact free measurements. Using thermocouples to measure the through-thickness temperature is only feasible for specific research purposes as they need to be embedded in the thermoplastic sheet. Since the embedded thermocouples cannot be removed without damaging the part, this method can be seen as a destructive measuring technique which is of course unacceptable in industrial applications. Contact free methods are based on intensity measurements of the emitted infrared radiation by the thermoplastic sheet using thermal cameras or pyrometers. Making an accurate temperature measure- 
ment based on IR radiation, is however complicated by different factors: unknown temperature dependent emissivity of the sheet material, the need to tune the measuring wavelength range of the sensor to the wavelength spectrum of the emission, directional dependency, difficulties to filter out the interfering reflectance, ... (Monteix et al., 2001; Haji and Spruiell, 1994a; Haji and Spruiell, 1994b). In practice, these artefacts can easily result in measurement errors up to $15^{\circ} \mathrm{C}$ (and sometimes more). For some complex products or materials, the forming range can be as small as $10^{\circ} \mathrm{C}$. The aforementioned errors are thus unacceptable. In addition, infrared measurements are considered to be surface measurements and are meaningless when evaluating the through-thickness temperature. For the purpose of the present study, accurate temperature measurements were performed with thermocouples.

Modelling the IR heating step of the thermoforming process has been the topic of different studies. A first approach was to apply a standard enclosure analysis to predict the IR heating of the sheet. (Brogan and Monaghan, 1996; Cunningham et al., 1997; Cunningham et al., 1998). Brogan and Monaghan (1996) eliminated the need for exact temperature settings of quartz tube heaters, by basing their model on the electrical power input to the heater elements. Cunningham et al. (1997) performed a study on contact, convective and radiative heat transfer to heat up thermoplastic sheets. The developed enclosure methods showed reasonable accuracy compared to experimental temperature measurements performed on thermoplastic sheets during heating. One year later, Cunningham et al. (1998) added a sheet transfer step from the heating position to forming positions to their model.

The most widespread method to model the heating of a thermoplastic sheet is however the explicit or implicit finite Difference method (Holman, 1997, Gauthier et al., 2005). This method is based on the basic heat balance equation and allows adding additional thermal effects (e.g. temperature dependent boundary conditions or material properties) when necessary.

To increase the accuracy of the through-thickness temperature prediction, the calculations need to take into account that the incident IR heating sheet is not completely absorbed at its surface but partially penetrates inside the sheet. This results in a rather volumetric heating compared to a pure surface heating (Throne, 1999). Based on the law of Lambert-Beer, the optical penetration depth is defined as the distance from the irradiated surface where the radiation intensity has decreased to $1 / \mathrm{e}$ $(\approx 36.8 \%$ ) of the intensity at the surface (Puehringer et al., 2011; Puehringer et al., 2013). The higher the optical penetration depth is, the more radiation is absorbed deeper in the volume of the sheet, thus improving the uniformity of the through-thickness temperature distribution without increasing the heating time. Gauthier et al. (2005) proved that the implementation of the penetration depth in a model to predict the IR heating of thermoplastic sheet improves the accuracy of the model when comparing results to experimental data.

Finite element thermal modelling as an alternative for finite difference was demonstrated by Erchiqui et al. (2007) by making use of the Galerkin method. Although feasible, the use of finite elements in thermoforming is most often applied when a subsequent mechanical deformation analysis of the thermoplastic sheet is performed (Erchiqui et al., 2007; Labeas et al., 2008; Cosson et al., 2011).
Cosson et al. (2011) used the ray tracing method as an alternative for the use of view factors to predict sheet heating Although this method is more computer intensive compared to the calculation of view factors, it describes the physical phenomena taking place in infrared heating in a more realistic way.

More recently the use of virtual sensors and the use of model predictive controllers are explored. The main goal of these methods is to estimate the temperature distribution of the whole sheet based on known physical models, instead of estimating or directly measuring temperatures at certain points (Chy and Boulet, 2010). The potential of this type of controllers has been proven in numerical studies but the high computational time or power, which is still required for this type of systems, obstructs an immediate industrial application (Chy and Boulet, 2011a; 2011b; 2012; Modirnia and Boulet, 2013).

Next to the development of numerical models to describe and predict the heating of thermoplastic sheets, the need for reliable material data and an accurate process description cannot be underestimated (Cosson et al. 2011). Density, heat conductivity and specific heat capacity are the key parameters to model the thermal behaviour of a thermoplastic material (Dos Santos, 2005).

IR heating is a complex physical process in which multiple parameters are involved. Moreover, most of these parameters are rarely readily available. The type of heating element determines the wavelength at which energy is transferred to the thermoplastic sheet. On its turn the sheet has its own wavelength dependent absorption spectrum (Schmidt et al., 2003). On top of this, the IR radiation might be absorbed, transmitted or reflected by both the thermoplastic sheet and the surrounding equipment (Girard et al., 2004; Throne, 1999; Chy and Boulet, 2011a; Khan et al., 2012). Next to the IR radiative heating, convective heat transfer at the surface will occur simultaneously. Taking this convective component into account is often complicated by the varying air temperature as a function of time and location (Yousefi et al. 2002; Chy and Boulet, 2011a). Finally the heating strategy itself will influence the heating of the thermoplastic sheet. On most thermoforming installations, heating can be done one-sided or two-sided and using a constant, two-step or pulsed setting of the heating elements (Progelhof et al., 1973; Sweeney et al.; 1995; Buffel and Desplentere, 2016). All of this leads to an equipment dependent heating of a thermoplastic sheet.

As a solution for the aforementioned issues, the present study proposes a method in which the heating equipment is fully characterized on site. In this way all parameters influencing the heating of a thermoplastic sheet are taken into account. A finite difference model is developed to reverse engineer the heating characteristics of the equipment. This results in a dataset which is used to optimize the heating strategy, and in turn can be implemented on the real equipment. When applied to different sheet materials and thicknesses, the optimization script will result in less trial and error iterations at start-up times and thus lower costs.

\section{Experimental}

Temperatures vs. time measurements of the heated material were performed by means of an instrumented silicone sheet in 
which 15 thermocouples (type $\mathrm{K}$ ) are embedded. The thermocouples are located at five different in-plane positions with on each location three thermocouples through-thickness. The through-thickness thermocouples are shifted $5 \mathrm{~mm}$ in-plane to reduce the interaction on each other (Fig. 1). The total thickness of the silicone sheet is $3.9 \mathrm{~mm}$. This sheet allows monitoring the top and bottom as well as the core temperature as a function of time and location. In the present study, only the through-thickness temperature distribution of the thermocouples at the center of the sheet is considered. The density, thermal conductivity and specific heat capacity of the silicone sheet at room temperature are respectively $1380 \mathrm{~kg} / \mathrm{m}^{3}$, $0.18 \mathrm{~W} / \mathrm{mK}$ and $1330 \mathrm{~J} / \mathrm{kgK}$.

Temperature measurements were conducted on two different thermoforming installations: a thermoformer (U8, Geiss, Sesslach, Germany) equipped with halogen heating elements (Philips, Pont à Mousson, France) and a thermoformer (UA 200ED, Illig, Heilbronn, Germany) which uses ceramic heating elements made by Elstein, Northeim, Germany. The former possesses a total power of $30.9 \mathrm{~kW}$ on a surface of $0.6 \mathrm{~m}^{2}$; resulting in radiative heat flux of $51.5 \mathrm{~kW} / \mathrm{m}^{2}$ on top and bottom the heating plane. The latter has an installed flux of $28 \mathrm{~kW} / \mathrm{m}^{2}$ in the upper heater, while the bottom heater has a maximum flux of $14 \mathrm{~kW} / \mathrm{m}^{2}$.

In order to completely characterize a thermoforming installation a series of heating experiments was performed. In case of halogen heating elements different percentages of the maximum power were set in steps of $10 \%$ : 40-50-60-70-80-90$100 \%$. The lower boundary of $40 \%$ was selected to avoid damage of the halogen heating elements. In the case of ceramic heater elements the temperature of the elements was adjusted in steps of $50^{\circ} \mathrm{C}: 250-300-350-400-450{ }^{\circ} \mathrm{C}$. These temperature settings are measured by an internal thermocouple in the ceramic heaters and maintained by the control system of the thermoformer. The temperature measurements of the instrumented silicone sheet are used as input for the optimization of the heating strategy of a thermoplastic sheet in thermoforming. Figure 2 schematizes the optimization workflow.

After the experimental temperature measurements are performed, a reverse engineering approach is used to characterize the heating equipment. This approach results in a data set describing the heating characteristics of the equipment in an in- dustrial environment and thus taking all parameters that could possibly influence the heating into account. In the case where halogen heater elements are used, the thermal efficiency with respect to the installed electrical power can be determined as well. Once these equipment dependent parameters are determined, the optimal heating settings for a given thermoplastic sheet can be predicted, provided the density, thermal conductivity, specific heat capacity of the material and the required temperature forming range are known.

\section{Modelling}

The standard heat balance differential equation is used to model the heating of a thermoplastic sheet. In this model conductive, convective and radiative heat transfer are taken into account (Eq. 1).

$\frac{\mathrm{dQ}_{\text {in }}}{\mathrm{dx}}-\frac{\mathrm{dQ}_{\text {out }}}{\mathrm{dx}}=\mathrm{mC}_{\mathrm{p}} \frac{\mathrm{d} \theta}{\mathrm{dt}}$.

As the present study focuses on an optimization of the throughthickness temperature distribution, only the thickness direction, $\mathrm{x}$, is considered. In Eq. $1 \mathrm{Q}$ denotes the heat flow, $\mathrm{m}$ is the mass, $\mathrm{C}_{\mathrm{p}}$ is the specific heat capacity, $\theta$ is the temperature in ${ }^{\circ} \mathrm{C}$ and $\mathrm{t}$ is the time variable. The heat balance equation is

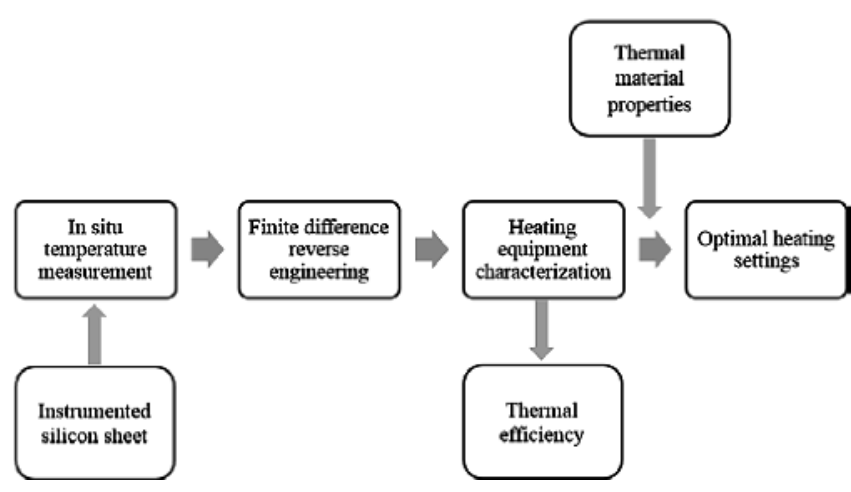

Fig. 2. Workflow for the characterization of the heating equipment and the determination of the optimal heating settings
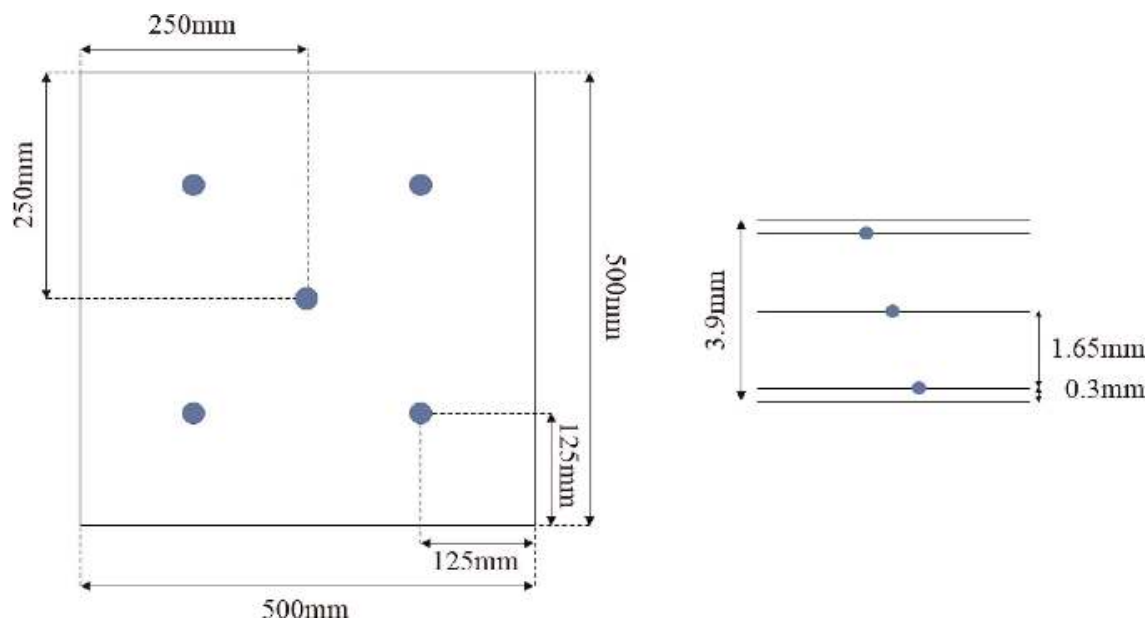

Fig. 1. Schematic representation of the instrumented silicone sheet: top view indicating the five different measurement locations (left); cross section indicating the position of the thermocouples through thickness (right) 
solved by means of the explicit finite difference method (Holman, 1997). The discretization of the sheet thickness, $\delta$, and size of the time step, $\Delta \mathrm{t}$, are determined by the Courant-Friedrichs-Lewy condition (Eq. 2) (Courant et al., 1967):

CFL value $=\frac{a \cdot \Delta \mathrm{t}}{\delta^{2}}<0,5$.

In Eq. $2 a$ is equal to the thermal diffusivity of the thermoplastic material. Equation 3 describes the boundary condition applied to the outer surfaces. In case the surface is exposed to incident radiation, a specific flux value is assigned to $\mathrm{q}_{\mathrm{rad}}$. When the surface is only experiencing convection to the environment $\mathrm{q}_{\mathrm{rad}}$ is set to zero.

$\mathrm{Q}_{\text {in }}=\alpha \mathrm{A}\left(\theta_{\infty}-\theta_{\mathrm{i}}\right)+\mathrm{q}_{\mathrm{rad}} \mathrm{A}$.

In Eq. $3 \alpha$ represents the convective heat transfer coefficient, $\theta_{\infty}$ the air temperature of the environment, $\theta_{\mathrm{i}}$ the surface temperature of the thermoplastic sheet, A the exposed surface area and $\mathrm{q}_{\mathrm{rad}}$ the radiative heat flux absorbed by the thermoplastic sheet. Due to the high filament temperature of the halogen element compared to the sheet temperature, the net radiative heat flux is approximated by a constant value in case of halogen heater elements. In case heater element temperatures are lower (e.g. quartz or ceramic elements), the radiative net heat flux decreases as the sheet temperature increases. In this case the net heat flux is calculated using the standard Stephan-Boltzmann equation. The through-thickness transport of heat in the sheet is modelled using Fourier's law (Eq. 4).

$\mathrm{Q}_{\text {conduction }}=-\lambda \mathrm{A} \frac{\mathrm{dQ}}{\mathrm{dx}}$.

In Eq. $4 \lambda$ represents the thermal conductivity of the thermoplastic material.

By combining Eqs. 1, 3, and 4 in a finite difference algorithm, a solution can be found for the through-thickness temperature distribution as a function of time. Since thermoplastic materials are semi-transparent for the incident radiative energy (Throne, 2016) the heat flux $\mathrm{q}_{\mathrm{rad}}$ is not only absorbed at the surface, but is also partially absorbed in the core of the sheet. This is taken into account by implementing the optical penetration depth, dp, (Puehringer et al., 2011; Puehringer et al., 2013) in the described model. The higher the optical penetration depth is, the deeper the IR radiation will penetrate the thermoplastic sheet resulting in a more volumetric heating compared to a pure surface heating effect. This parameter depends on the type of material of the sheet as well as the wavelength of the applied IR radiation. Since the latter is influenced by the temperature of the heater elements, the penetration depth will be influenced by the type and setting of heater elements used. Due to the high filament temperatures and shorter wavelength of the emitted radiation, halogen elements are known to give a higher penetration depth compared to quartz or ceramic elements.

The method proposed by Gauthier et al. (2005) was used to model the penetration of the radiative heat flux and determine the through-thickness radiative heat flux distribution (Eq. 5 and 6).

$\beta=1-\mathrm{e}^{\Delta \mathrm{x} / \mathrm{d}_{\mathrm{p}}}$,

$\mathrm{q}_{\mathrm{i}}=\beta(1-\beta)^{\mathrm{i}-2}(1-\beta) \mathrm{q}_{\mathrm{rad}}$.
The term $\mathrm{q}_{\mathrm{i}}$ was added to the heat balance for each layer (i) through the thickness of the sheet.

The described model framework is applicable for all types of heater elements. In the case of halogen heater elements, an additional effect is taken into account. These elements are typically switched on and off in every cycle to reduce energy costs and to protect the halogen elements. When the heater elements are switched on, they require a short period of time to reach a constant temperature due to thermal inertia of the filament. This, experimentally observed, time delay was implemented in the model with a time constant $\tau$ in a first order system (Eq. 7):

$\mathrm{q}(\mathrm{t})=\mathrm{q}_{\mathrm{rad}} \mathrm{e}^{-\tau / \mathrm{t}}$.

Combining all previous equations, a one-dimensional explicit finite difference algorithm was developed based on one-dimensional heat transport by means of convection, conduction and radiation taking both radiative penetration and halogen heater element start-up delay into account. As mentioned previously, in the developed model, the thermoplastic sheet is divided into multiple layers with thickness $\delta$, which were numbered from 1 up to $n$. The elaborated equations in the algorithm for the outer surfaces $(i=1, n)$ and the inner layers are respectively presented in Eqs. 8, 9 and 10.

$$
\begin{gathered}
\theta_{i}^{\mathrm{t}+1}=\theta_{\mathrm{i}}^{\mathrm{t}}+\frac{\Delta \mathrm{t}}{\rho \mathrm{C}_{\mathrm{p}} \delta}\left[\mathrm{q}_{\mathrm{rad}}(\mathrm{t}, \mathrm{i})+\frac{\lambda}{\delta}\left(\theta_{\mathrm{i}+1}^{\mathrm{t}}-\theta_{\mathrm{i}}^{\mathrm{t}}\right)-\alpha\left(\theta_{\mathrm{i}}^{\mathrm{t}}-\theta_{\infty}^{\mathrm{t}}\right)\right] \\
\text { with } \mathrm{i}=1, \\
\theta_{\mathrm{i}}^{\mathrm{t}+1}=\theta_{\mathrm{i}}^{\mathrm{t}}+\mathrm{q}_{\mathrm{rad}}(\mathrm{t}, \mathrm{i}) \frac{\Delta \mathrm{t}}{\rho \mathrm{C}_{\mathrm{p}} \delta}+\mathrm{a} \frac{\Delta \mathrm{t}}{\delta^{2}}\left[\theta_{\mathrm{i}-1}^{\mathrm{t}}+\theta_{\mathrm{i}+1}^{\mathrm{t}}-2 \theta_{\mathrm{i}}^{\mathrm{t}}\right] \\
\text { with } \mathrm{i}=] 1, \mathrm{n}[
\end{gathered}
$$

$\theta_{\mathrm{n}}^{\mathrm{t}+1}=\theta_{\mathrm{n}}^{\mathrm{t}}+\frac{\Delta \mathrm{t}}{\rho \mathrm{C}_{\mathrm{p}} \delta}\left[\mathrm{q}_{\mathrm{rad}}(\mathrm{t}, \mathrm{n})+\frac{\lambda}{\delta}\left(\theta_{\mathrm{n}-1}^{\mathrm{t}}-\theta_{\mathrm{n}}^{\mathrm{t}}\right)-\alpha\left(\theta_{\mathrm{n}}^{\mathrm{t}}-\theta_{\infty}^{\mathrm{t}}\right)\right]$ with $\mathrm{i}=\mathrm{n}$.

These equations are solved for each time step t until the required through thickness temperature distribution is reached. As indicated in these equations the temperature of the surrounding air and the applied radiative heat flux can be a function of time. The latter is also depending on the through thickness position and time with respect to switching on the halogen elements through Eqs. 5, 6 and 7.

In the present study, this model was used to characterize the thermoforming heating equipment by means of reverse engineering the different parameters based on experimental measurements.

Next to the characterization of the equipment, the optimal heating setting for a given heater bank and material can be determined. For this purpose an iterative algorithm was implemented on the same model. This iteration method solves the equations to find the fastest heating method to obtain a through-thickness temperature distribution within the desired temperature forming range. In the iterative process the sheet is heated until the exposed surface reaches the upper boundary of the forming temperature range. Next, a check is performed on the coldest position through the thickness of the sheet. In case the temperature at this position did not reach the lower 
boundary of the pre-defined forming temperature range, the heating power is decreased by $10 \%$ and the process is repeated. The optimal heating parameters are defined as those for which the coldest position has also reached the forming range. The procedure is applicable for both single and two sided heating.

In most thermoforming installations, there is a short timespan between the end of the heating stage and the start of the actual forming, caused by the retraction of the heaters and the rising of the mold. In this timespan the outer surfaces of the sheet will cool down by convective heat transfer while the core will first continue to heat up due to thermal inertia effects. When the considered material allows a small overshoot of the temperature forming range, these thermal effects allow to to further decrease the required heating time. This effect is integrated in the presented model.

\section{Results and Discussion}

In this section results will be shown for the workflow presented in Fig. 2. As mentioned before the whole workflow is applicable to all types of heater elements and single or two sided heating. In the following paragraphs the results will be shown for one sided heating with halogen heater elements. Results of the experimental sheet temperature measurements, the reverse engineering step and determination of the thermal efficiency are presented. As a proof of concept, the optimal heating settings are determined for one-sided heating with ceramic heater elements and validated for a light cover application produced out of a $3 \mathrm{~mm}$ thick high impact polystyrene material.

\subsection{In situ Temperature Measurements}

The experimental heating curves measured with the instrumented silicone sheet for 30, 50, 70 and $100 \%$ of the maximum halogen heater power are shown in Fig. 3. The subsequent cooling down of the sheet was not taken into account in the present model. In order to prevent sagging of the instrumented silicone sheet, it was heated on one side up to a maximum temperature of $90^{\circ} \mathrm{C}$. The higher the applied heating power, the faster the heated side reaches the maximum temperature. At the same time however, the through-thickness temperature difference increases. This poses an upper limit on the maximum achievable heating rate, as the sheet should reach temperatures within the forming temperature range throughout the whole thickness.

Once the heater elements are switched off, an immediate drop in surface temperature on the heated side is observed. This is caused by convective heat transfer to the environment and conductive heat transfer towards the core of the thermoplastic sheet. At the opposite side and in the core of the sheet, the temperature keeps increasing for a short period of time before cooling down as well. The minimum temperature difference through the thickness of the sheet is observed at the moment when the opposite side reaches its maximum. If the temperature of the heated side would still be within the forming range at this point, this would be the best moment to form the product as the sheet will experience the least shear stresses between layers of different temperatures.

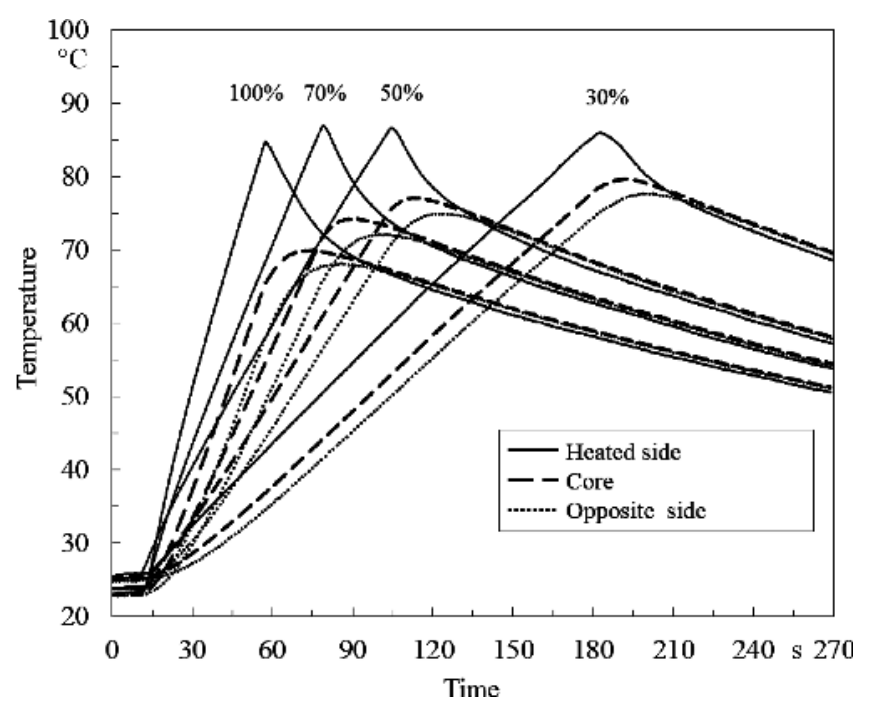

Fig. 3. Experimentally determined heating curves for one sided heating at different power settings (expressed in \%) of the halogen heating elements. The temperatures are monitored with thermocouples embedded in the instrumented silicone sheet

\subsection{Determination of the Equipment Specific Heating Parameters}

Experimentally measured heating curves were used in the reverse engineering step to characterize the heating equipment of the thermoforming installation. The different parameters taken into account in the procedure are listed in Table 1.

In order to obtain a complete characterization of the equipment, the optimal parameter set was determined for different heat fluxes emitted by the heater elements at different settings. In this procedure the experimental temperature curves were used as a reference to determine the best fitting parameters. It is important to note that the optical penetration depth is both wavelength and material dependent. By using this parameter in the fitting routine, only the wavelength dependency and not the material dependency is taken into account. This approach was selected in order to obtain an easily applicable method at the industrial scale. Determining the optical penetration depth for each material type and grade is a time consuming task reserved for specialized laboratories (Puehringer et al., 2013). An example of this is shown in Fig. 4 where the experimental heating curves for single sided heating at $80 \%$ and at $50 \%$ of the maximum power are approximated by the finite difference model described earlier (cfr. section 3 Modelling). Based on the deviation between experimental and modelled data and the final slope of the modelled heating curves, a very good agreement between the model and experimental data can be obtained. It is important to note that for each heater setting the same value for each of the parameters is applied for all three curves. The fitted values change however for each heater setting.

The result of this reverse engineering step is a dataset in which each parameter of Table 1 is described by a single value per heater setting. This dataset is a complete characterization of the heating equipment in its own environment taking the local influencing parameters into account. 


\subsection{Thermal Efficiency of the Halogen Heating Equipment}

In practice, the amount of power emitted by halogen heater elements is determined by multiplying the selected power percentage by the maximum power of the heaters. Moreover, the emitted heat flux is considered to be constant because the filament temperature $(>1400 \mathrm{~K})$ is an order of magnitude higher compared to the sheet temperature. When comparing the emitted heat flux to the net absorbed heat flux, determined in the reverse engineering procedure, the thermal efficiency of the heating equipment can be calculated.

The thermal efficiency for one sided heating of the used halogen heater elements is defined as the ratio of the absorbed to the emitted heat flux and is presented in Fig. 5 as the slope of the regression line through the different measuring points. A thermal efficiency value of $19 \%$ was obtained. The high value of the correlation coefficient of the linear regression indicates a nearly constant thermal efficiency in the tested range of the halogen heater elements.

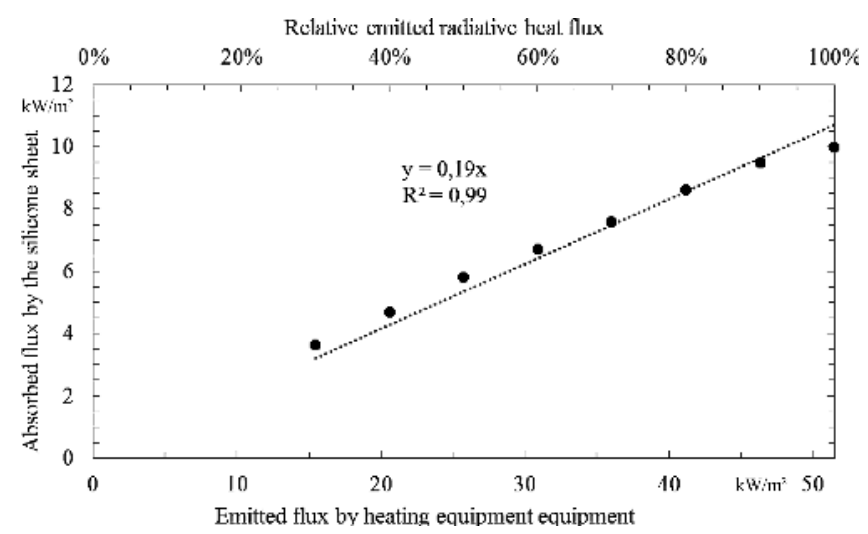

Fig. 5. Comparison of the emitted heat flux to the absorbed heat flux allowing the determination of the thermal efficiency of the heating equipment

\begin{tabular}{|c|c|c|}
\hline Parameter & $\begin{array}{c}\text { Halogen } \\
\text { heater elements }\end{array}$ & $\begin{array}{c}\text { Ceramic/Quartz } \\
\text { heater elements }\end{array}$ \\
\hline Constant radiative heat flux & $\mathrm{V}$ & - \\
Temperature of the heater elements & - & $\mathrm{V}$ \\
Radiative number (Stephan-Boltzmann Law) & $\mathrm{V}$ \\
Convective $\alpha$ top side & $\mathrm{V}$ & $\mathrm{V}$ \\
Convective $\alpha$ bottom side & $\mathrm{V}$ & $\mathrm{V}$ \\
Environment temperature top side & $\mathrm{V}$ & $\mathrm{V}$ \\
Environment temperature bottom side & $\mathrm{V}$ & $\mathrm{V}$ \\
Optical penetration depth & $\mathrm{V}$ & - \\
Halogen element start-up delay time & & \\
\hline
\end{tabular}

Table 1. Used parameters for the reverse engineering procedure in case of heating with halogen, ceramic and quartz heater elements
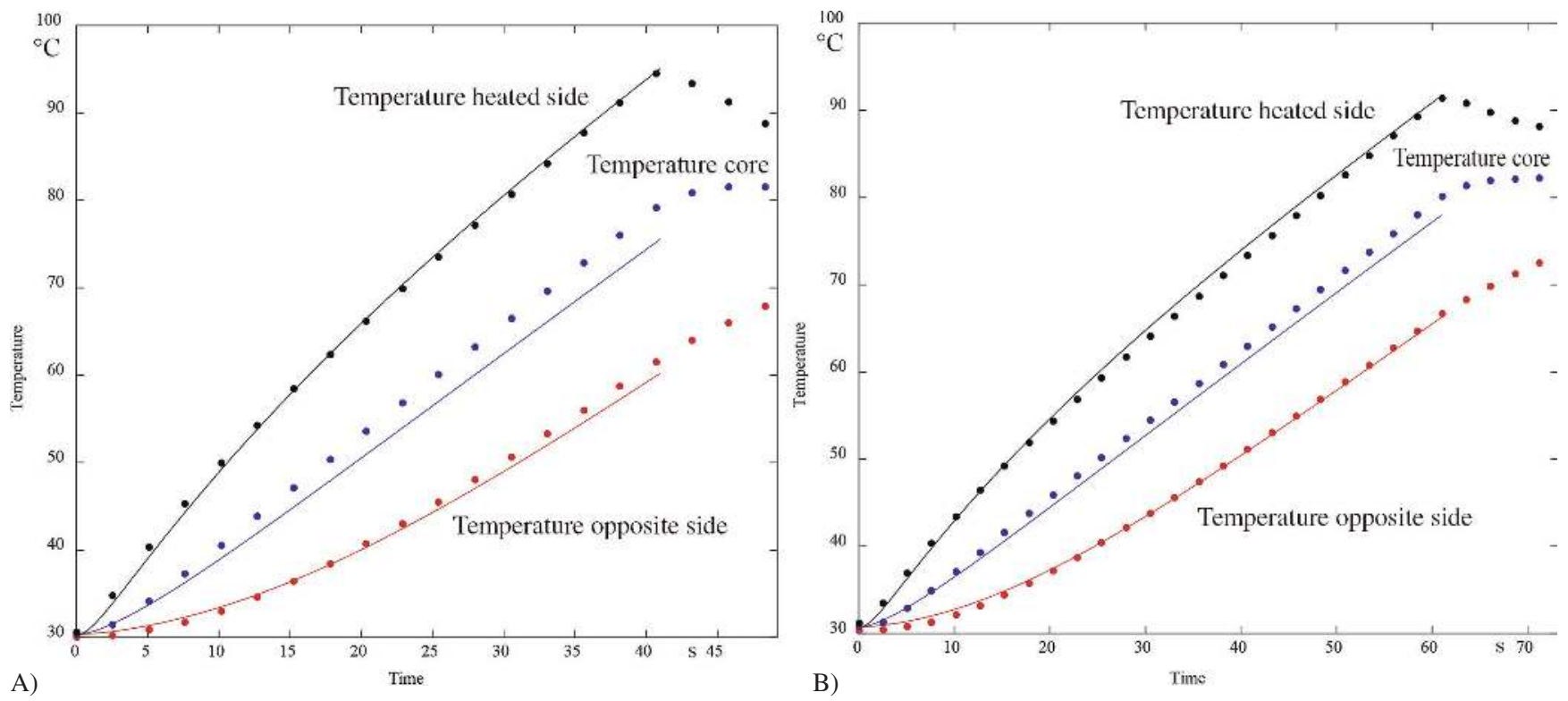

Fig. 4. Comparison between experimental heating data (dots) and reverse engineered curves (solid lines) for heater settings of $80 \%$ (A) and $50 \%$ $(B)$ of the maximum available heating power. The reverse engineering step allows the determination of the parameters which describe the heating equipment the best 


\subsection{Optimized Heating Settings for a Light Cover Application}

The obtained dataset describing the heating equipment was used to determine the optimal heating settings for a high impact polystyrene (HIPS) sheet with a thickness of $3 \mathrm{~mm}$. The sheet has a density of $1050 \mathrm{~kg} / \mathrm{m}^{3}$ and a thermal conductivity of $0.15 \mathrm{~W} / \mathrm{mK}$. The conductivity was experimentally measured by means of the Modified Transient Plate Source (MTPS) method with a C-therm Thermal conductivity Analyzer. The temperature dependent specific heat capacity was measured with a TA instruments Q200 MDSC device.
The goal of the optimization procedure is to find the optimal heating settings to form a light cover (Fig. 6) in an aluminum mold (negative forming). The mold was previously produced by Single Point Incremental Forming (SPIF). At the top, the mold cavity has a diameter of $390 \mathrm{~mm}$ which narrows towards a diameter of $180 \mathrm{~mm}$ at the base over a vertical distance of $275 \mathrm{~mm}$.

Based on the data available in Throne (1996) the temperature forming range of the material was between $130^{\circ} \mathrm{C}$ up to $160^{\circ} \mathrm{C}$ and is indicated in Fig. 7 by the grey hatched area on the graph. By applying the optimization algorithm described in section 3, the optimal temperature setting for the ceramic heater and the required heating time are respectively $315^{\circ} \mathrm{C}$
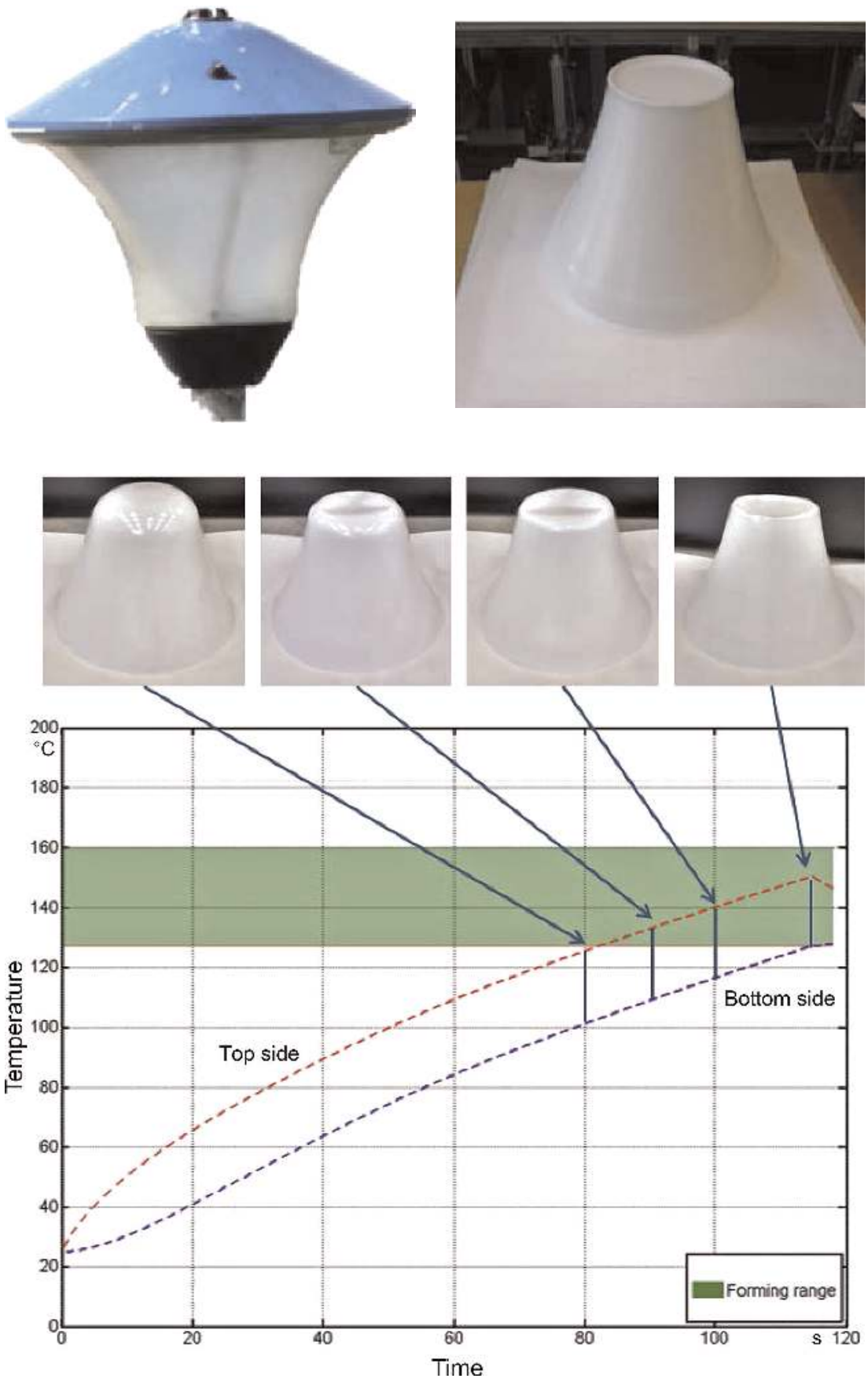

Fig. 6. Light cover application (left), thermoformed part to be used in the light cover (right)
Fig. 7. Optimal heating curve for the HIPS sheet. When the optimal heating time is not respected, and forming of the product is attempted at shorter times, the product is not completely formed and has to be considered as scrap 
and $116 \mathrm{~s}$. The optimal heating curve for the heated and opposite side of the HIPS sheet is shown in Fig. 7. Note that the curve for the heated side of the sheet does not reach the upper boundary of the forming range. This is because the highest temperature of the sheet is not at the outer surface of the heated side due to convective heat losses. The highest temperature occurs at a short distance underneath the surface and reaches the upper limit of the forming range.

In order to validate the proposed optimal heating settings, four runs were tested in which the sheet was heated with identical heater settings but different heating times. The light cover was formed after heating times of 80, 90, 100 and $116 \mathrm{~s}$. The resulting products of these runs are presented in the images on Fig. 7. The product could only be completely formed after the heating time was set to the value $115 \mathrm{~s}$ as, proposed by the optimization algorithm. When the light cover was formed at shorter times, the HIPS sheet was not at the desired temperature forming range across the complete thickness, resulting in an incomplete forming of the product. This is evidenced by the pictures in Fig. 7 in which the sharp edges at the top of the product are only formed when a heating time of $115 \mathrm{~s}$ is applied. This successfully validates the results of the optimization procedure. Moreover it proves the validity of the characterization procedure in which only the wavelength dependency of the penetration depth is taken into account.

\section{Conclusions}

The present study has developed an on-site characterization method for the heating equipment in thermoforming. The method can be used for all different types of heating elements, it takes local interfering effects into account and it can easily be implemented in an industrial environment. Moreover in case of halogen heating elements, the thermal efficiency of the equipment can be estimated.

An optimization algorithm was developed to determine the optimal heater setting of the equipment (heater setting and heating time) for different materials and sheet thicknesses. The algorithm was successfully validated through the experimental forming of a product. Due to varying boundary conditions in an industrial environment, it is expected that the optimization scheme in practice will yield a well-considered first value for the machine setting during process start-up. In comparison to the currently applied trial and error method, this will result in lower start-up times and costs as well as less dependency on the experience of the machine operator.

A short delay time prior to the actual forming step is used to obtain a more homogeneous through thickness temperature distribution of the thermoplastic sheet. In practice this delay time can simultaneously be used to perform necessary machine movements like sheet transfer or displacements of molds and/ or plug assists.

Future work will look into the optimization of two-step and pulsed heating methods as well as further investigate the difference in material properties of the thermoplastic sheet material and silicone material of the instrumented sheet. Especially the value of the optical penetration depth for different thermoplastic materials with respect to the silicone material is of high interest for future developments.

\section{References}

Brogan, M. T., Monaghan, P. F., "Thermal Simulation of Quartz Tube Infra-Red Heaters Used in the Processing of Thermoplastic Composites", Composites Part A, 27A, 301-306 (1996), DOI: $10.1016 / 1359-835 X(95) 00056-8$

Buffel, B., Desplentere, F., "A Numerical Study on Different Heating Strategies in Thermoforming of Thermoplastic Sheets", Proceedings of the Polymer Moulds and Innovation Conference, Ghent, 221-226 (2016)

Cosson, B., Schmidt, F., Le Maoult, Y. and Bordival, M., "Infrared Heating Stage Simulation of Semi-Transparent Media (PET) Using Ray Tracing Method", Int. J. Mater. Form., 4, 1-10 (2011), DOI:10.1007/s12289-010-0985-8

Courant, R., Friedrichs, K. and Lewy, H., "On the Partial Difference Equations of Mathematical Physics", IBM J. Res. Develop., 11, 215-234 (1967), DOI:10.1147/rd.112.0215

Chy, M. I., Boulet, B.: A New Method for Estimation and Control of Temperature Profile Over a Sheet in Thermoforming Process, IEEE Publications, Houston (2010)

Chy, M. I., Boulet, B.: Development of An Improved Mathematical Model of the Heating Phase of Thermoforming Process, IEEE Publications, Orlando (2011a)

Chy, M. I., Boulet, B., "A Model Predictive Controller of Plastic Sheet Temperature for A Thermoforming Process", Proceedings of the American Control Conference, San Francisco, 4410-4415 (2011b), DOI:10.1109/acc.2011.5991551

Chy, M. I., Boulet, B., "Iterative Learning Model Predictive Controller of Plastic Sheet Temperature for A Thermoforming Sheet", Proceedings of the American Control Conference, Montreal, 627-633 (2012), DOI:10.1109/acc.2012.6315521

Cunningham, J. E., Monaghan, P. F., Brogan M. T. and Cassidy, S. F., "Modelling of Pre-Heating of Flat Panels Prior to Press Forming", Composites Part A, 28A, 17-24 (1997), DOI:10.1016/S1359-835X(96)00089-9

Cunningham, J. E., Monaghan, P. F. and Brogan M. T., "Prediction of the Temperature Profile within Composite Sheets during Pre-Heating", Composites Part A, 29A, 51-61 (1998), DOI: $10.1016 / \mathrm{S} 1359-835 \mathrm{X}(97) 00031-6$

Dos Santos, W. N., "Thermal Properties of Melt Polymers by the Hot Wire Technique", Polym. Test., 24, 932-941 (2005), DOI:10.1016/j.polymertesting.2005.06.001

Duarte, F. M., Covas, J. A., "On the Use of the Heating Stage to Control the Thickness Distribution in Thermoformed Parts", Int. Polym. Proc., 19, 186-198 (2004), DOI:10.3139/217.1821

Erchiqui, F., Souli, M. and Yedder, R. B., "Nonisothermal Finite-Element Analysis of Thermoforming of Polyethylene Terephthalate Sheet: Incomplete Effect of Forming Stage", Polym. Eng. Sci., 47, 2129-2144 (2007)

Gauthier, G., Mark, A., Boulet, B., Haurani, A., Girard, P. and Diraddo, R., "A New Absorption Based Model for Sheet Reheat in Thermoforming", SPE ANTEC Tech. Papers, 353-357 (2005)

Gilham, E., "Lean Manufacturing", SPE European Thermoforming Division Conference, Sitges (2016)

Girard, P., Di Raddo, R., Thomson, V. and Boulet, B.: Advanced InCycle and Cycle to Cycle Online Adaptive Control for Thermoforming of Large Thermoplastic Sheets, SAE International, NCR Publications, (2004), DOI:10.4271/2005-01-1520

Haji, N., Spruiell, J. E., "Radiation Pyrometry on Semitransparent Sheets. I: Gray Media", Polym. Eng. Sci., 34, 116-121 (1994a), DOI: $10.1002 /$ pen.760340207

Haji, N., Spruiell, J. E., "Radiation Pyrometry on Semitransparent Sheets. II: Media with Wavelength Dependent Absorption Coefficient", Polym. Eng. Sci., 34, 122-127 (1994b), DOI: $10.1002 /$ pen.760340208

Holman, J. L.: Heat Transfer, $8^{\text {th }}$ Edition, McGraw-Hill, New York (1997)

Khan, S. A., Girard, P., Bhuiyan, N. and Thomson, V., "Improved Mathematical Modeling for the Sheet Reheat Phase during Thermoforming", Polym. Eng. Sci., 52, 625-636 (2012)

Labeas, G. N., Watiti, V. B. and Katsiropoulos, C. V., "Thermomechanical Simulation of Infrared Heating Diaphragm Forming Process 
for Thermoplastic Parts", J. Thermoplast. Compos. Mater., 21, 353-370 (1998), DOI:10.1177/0892705708089480

Lee, J. K., Virkler, T. L. and Scott, C. E., "Effects of Rheological Properties and Processing Parameters on ABS Thermoforming", Polym. Eng. Sci., 41, 240-261 (2001a), DOI:10.1002/pen.10725

Lee, J. K., Virkler, T. L. and Scott, C. E., "Influence of Initial Sheet Temperature on ABS Thermoforming", Polym. Eng. Sci., 41, 1830-1844 (2001b), DOI:10.1002/pen.10880

Marathe, D., Rokade, D., Busher Azad, L., Jadhav, K., Mahajan, S., Ahmad, Z., Gupta, S., Kulkarni, S., Juvekar, V. and Lele, A., "Effect of Plug Temperature on the Strain and Thickness Distribution of Components Made by Plug Assist Thermoforming", Int. Polym. Proc., 31, 166-178 (2016), DOI:10.3139/217.3060

Modirnia, R., Boulet, B., "Model-Based Virtual Sensors and CoreTemperature Observers in Thermoforming Applications", IEEE Trans. Ind. App., 49, 721-730 (2013), DOI:10.1109/TIA.2013.2244544

Monteix, S., Schmidt, F., Le Maoult, Y., Ben Yedder, R., Diraddo, R. W. and Laroche, D., "Experimental Study and Numerical Simulation of Preform or Sheet Exposed to Infrared Radiative Heating", J. Mater. Process. Technol., 119, 90-97 (2001), DOI:10.1016/S0924-0136(01)00882-2

Progelhof, R. C., Quintiere, J., "Temperature Distribution in Semitransparent Plastic Sheets Exposed to Symmetric, Unsymmetric and Pulsed Radiant Heating and Surface Cooling", J. Appl. Polym. Sci., 17, 1227-1252 (1973), DOI:10.1002/app.1973.070170418

Puehringer, J. F., Zitzenbacher, G. and Spreitzer, C., "Study of Heat Absorption in the Thermoforming Process for Transparent and Filled Polystyrene", Proceedings of the $27^{\text {th }}$ PPS Conference, Marrakesh (2011), PMid:21155540

Puehringer, J. F., Zitzenbacher, G. and Spreitzer, C., "Study of Heat Absorption in Thermoforming for Transparent and Filled Polystyrene", Int. Polym. Proc., 18, 14-23 (2013), DOI:10.3139/217.2595

Schmidt, F. M., Le Maoult, Y. and Monteix, S., "Modelling of Infrared Heating of Thermoplastic Sheet Used in Thermoforming Process", J. Mater. Process. Technol., 143-144, 225-231 (2003)

Sweeney, G. J., Monaghan, P. F., Brogan, M. T. and Cassidy, S. F., "Reduction of Infra-Red Heating Cycle Time in Processing of Thermoplastic Composites Using Computer Modelling", Composites Manufacturing, 6, 255-252 (1995),

DOI:10.1016/0956-7143(95)95018-T

Throne, J. L.: Technology of Thermoforming, $1^{\text {st }}$ Edition, Hanser Gardner Publications, Munich (1996), PMid:8672655;

DOI:10.3139/9783446402478
Throne, J. L., "Heating Semitransparent Polymers in Thermoforming", Thermoforming Quarterly, 18, 7-12 (1999)

Van Mieghem, B.: An Intelligent Experimental Approach for the Optimization of the Process Parameters for the Thermoforming of Plastics and Composites, PhD Dissertation, KU Leuven Science Engineering and Technology, Leuven (2015)

Yousefi, A., Bendada, A. and Diraddo, R., "Improved Modeling for the Reheat Phase in Thermoforming through an Uncertainty Treatment of the Key Parameters", Polym. Eng. Sci., 42, 1115-1129 (2002), DOI:10.1002/pen.11016

\section{Acknowledgements}

The authors wish to thank the Flemish government and the industrial partners for the funding of the IWT TETRA 1301179 project. All results discussed in the present paper were obtained within this project.

Date received: October 03,2016

Date accepted: November 23, 2016

Bibliography

DOI 10.3139/217.3370

Intern. Polymer Processing

XXXII (2017) 3; page 378-386

(c) Carl Hanser Verlag GmbH \& Co. KG

ISSN 0930-777X 